\title{
Research Paper \\ Evaluating of the Pregnancy Rate of Freezing Embryo Transfer in the Presence or Absence of GnRH Agonist
}

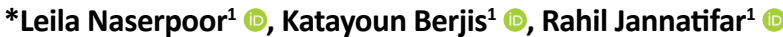

1. Department of Reproductive Biology, Academic Center for Education, Culture and Research, Qom, Iran.

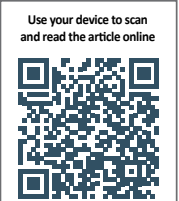

Citation: Naserpoor L, Berjis K, Jannatifar R. [Evaluating of the Pregnancy Rate of Freezing Embryo Transfer in the Presence or Absence of GnRH Agonist (Persian)]. Journal of Arak University of Medical Sciences (JAMS). 2021; 23(6):818-827. https:// doi.org/10.32598/JAMS.23.6.6127.1

doi' $h$ htps://doi.org/10.32598/JAMS.23.6.6127.1

Keywords:

Endometrium, GnRH, Embryo implantation, Embryo freezing

\section{A B STRACT}

Article Info:

Received: 01 Mar 2020

Accepted: 22 Jul 2020

Available Online: 01 Feb 2021

\section{I}

\section{Extended Abstract}

\section{Introduction}

n Assisted Reproductive Technologies (ARTs), if there are multiple embryos, the freezing method is used for excess embryos $[1,2]$. The golden time of implantation in the uterus is less than 48 hours $[3,4]$. The estrogen and progesterone hormones lead to embryo implantation by proliferation and changes in the endometrium of the uterus $[5,6]$. Various methods exist for preparing the endometrium to transfer the freezing embryo. The most prevalent approach is to exogenously use estrogen and progesterone with or without using GnRH agonists [7-10]. Considering the difficulty of injection and the cost of using GnRH agonists, researchers suggest using the method without GnRH [11]. Besides, it has a shorter treatment duration and presents a higher or equal success rate, compared to cycles with $\mathrm{GnRH}[12,13]$. Ac-

\section{* Corresponding Author:}

Leila Naserpoor

Address: Department of Reproductive Biology, Academic Center for Education, Qom, Iran

Tel: +98 (912) 3463922

E-mail: leilanasery48@gmail.com 
cordingly, the present study aimed to compare the results of using the two methods to prepare the endometrium.

\section{Materials and Methods}

This retrospective case-control study was performed on 146 infertile couples referring to the Infertility Treatment Center of the Academic Center for Education, Culture, and Research of Qom University, Qom, Iran from 2013 to 2015. The records of individuals who were candidates for the freezing embryo transfer cycle were reviewed in two treatment cycle groups of with and without GnRH agonist. In the first group, GnRH suppressors were used; $0.5 \mathrm{cc} /$ day of GnRH agonist (Buserline Cinagen, Iran) initiated from the 19th day of the previous menstrual cycle. Subsequently, with the patient's visit on the third day of menstruation, the agonist dose was reduced by half and estradiol 2 tablets daily were administered for 4 days. The next dose of estradiol was determined according to the conditions of the endometrium. When the endometrium was $7-9 \mathrm{~mm}$ thick, the patient was intramuscularly given 2 progesterone injections daily, then the fetus was transferred. The second group of patients was referred to the clinic on the third day of menstruation. Consequently, after daily ultrasound monitoring, $6 \mathrm{mg}$ estradiol valerate tablets were prescribed and the same procedure applied for the first group was performed for them. Clinical characteristics, implantation rate, pregnancy percentage (chemical \& clinical), and abortion rate were evaluated in the research groups. $\mathrm{P}<0.05$ was considered significant.

\section{Results}

The present study was conducted on 146 patients who used frozen embryos. The Independent Samples t-test revealed no significant difference between the treatment groups with and without $\mathrm{GnRH}$ agonist concerning the age of patients, the duration of infertility, endometrial thickness, and the number of embryos transferred in the research groups (Table 1). The Independent Samples t-test data indicated no significant difference in the mean age of patients between the study groups $(\mathrm{P}=0.3)$. The Independent Samples t-test results signified no significant difference in the mean duration of infertility between the study groups $(\mathrm{P}=0.4)$; the difference between the mean number of total embryos transferred was not significant between the study groups $(\mathrm{P}=0.6)$. The Independent Samples t-test data indicated no significant difference in the mean endometrial thickness between the research groups $(\mathrm{P}=0.841)$. Chi-squared test results revealed no significant difference in the frequency of embryo implants between the study groups treated with and without GnRH agonist $(\mathrm{P}=0.387)$. Chi-squared test data indicated no significant difference between the research groups in the number of chemical pregnancies $(\mathrm{P}=0.482)$. Chi-squared test results revealed no significant difference in the number of chemical pregnancies between the study groups treated with and without $\mathrm{GnRH}$ agonist $(\mathrm{P}=0.584)$. Eventually, Fisher's Exact test data demonstrated no significant difference in the frequency of abortion between the two There was no significant difference between the study groups $(\mathrm{P}=0.681)$ (Table 2$)$.

\section{Discussion and Conclusion}

Transfer cycles of frozen embryos require careful coordination between endometrial preparation and embryonic development [14]. Infertility treatment centers implement various approaches to prepare the endometrium for frozen embryo transfer [15]. The present research results reflected no significant difference between the study groups concerning demographic characteristics (female' age). Furthermore, no significant difference was observed in endometrial thickness on the day of embryo transfer and the number of embryos transferred between the study groups. The obtained data signified no significant difference between implantation rate and pregnancy rate [chemically positive ( $\beta$-HCG) \& clinical] in the study groups with or without GnRH agonist use. Considering the faster and easier effects as well as lower cost and drug consumption, and less ad-

Table 1. Comparing the clinical and baseline characteristics between GnRH agonist and non-GnRH agonist treatment groups

\begin{tabular}{|c|c|c|c|}
\hline \multirow{2}{*}{ Characteristic } & \multicolumn{2}{|c|}{ Mean \pm SD } & \multirow{2}{*}{$\mathbf{P}$} \\
\hline & GnRH Agonist Treatment Group (n: 74) & Non-GnRH Agonist Treatment Group (n: 74) & \\
\hline Age, $y$ & $36 \pm 7.6$ & $35.7 \pm 7.6$ & 0.321 \\
\hline The duration of infertility & $5.9 \pm 6.1$ & $5.7 \pm 6.1$ & 0.451 \\
\hline Endometrial thickness (mm) & $8.2 \pm 1.3$ & $8.4 \pm 1.7$ & 0.841 \\
\hline Number of transferred embryos ( $\mathrm{n}$ ) & $3 \pm 0.8$ & $3.1 \pm 0.6$ & 0.612 \\
\hline
\end{tabular}


Table 2. Pregnancy rate of frozen embryo transfer between GnRH agonist and non-GnRH agonist treatment groups

\begin{tabular}{|c|c|c|c|}
\hline \multirow{2}{*}{ Characteristic } & \multicolumn{2}{|c|}{ No. (\%) } & \multirow{2}{*}{$\mathbf{P}$} \\
\hline & GnRH Agonist Treatment Group & Non-GnRH Agonist Treatment Group & \\
\hline Implantation rate & $23(24.7)$ & $28(36.1)$ & 0.387 \\
\hline Chemical-based pregnancy & $22(221)$ & $26(30.7)$ & 0.482 \\
\hline Clinical-based pregnancy & $19(21.3)$ & $22(26.1)$ & 0.582 \\
\hline Abortion rate & $2(1.1)$ & $4(3.6)$ & 0.681 \\
\hline
\end{tabular}

Journal of
Arak University of Medical Sciences

verse effects generated by the medications, and of course, greater satisfaction among patients not using $\mathrm{GnRH}$, cycles without GnRH agonists cycles are more feasible and faster. They also provide a better estimate of the day of transfer and reduce costs. Furthermore, several studies applied different GnRH types and provided no evidence that their effects on pregnancy $[16,17]$. The incidence of abortion before 6 weeks was similar in both study groups; in both research groups, there was also no significant difference before 20 weeks. Our results were in line with those of a study conducted in 2014 [18] reporting no significant difference in the rate of abortion in both groups.

In this study, pregnancy outcome was similar in both study groups; thus, this method is recommended as an endometrial preparation without GnRH agonist.

\section{Ethical Considerations}

\section{Compliance with ethical guidelines}

This study was approved by the Research Ethics Committee of the Academic Center for Education, Culture, and Research of Mashhad (Code: IR.ACECR.JDM. REC.1398.001).

\section{Funding}

This study was funded by the Academic Center for Education, Culture, and Research of Qom, Qom.

\section{Authors' contributions}

Conceptualization: Leila Naserpour; Research and sampling: Katayoun Borjis, Leila Naserpour; Data analysis: Raheel Janatifar, Leila Naserpour.

\section{Conflicts of interest}

The authors declared no conflicts of interest.

\section{Acknowledgements}

We want to thank The Research Deputy of the Academic Center for Education, Culture, and Research of Qom for their cooperation in conducting this project. 
ارزيابى ميزٔان حاملكَى حاصل از انثقال جنين هاي فريزّى در حضور و عدم حضور آتوثيست GnRH

"ليلا ناصريور' ه. كتايون برجيس' ه. راحيل جنتىفر' هـ

ا. كروه يُؤهشى بيولوزى توليد مثل، جهاد دانشكاهى، قمه ايران.

\begin{abstract}
(1)

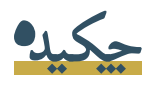

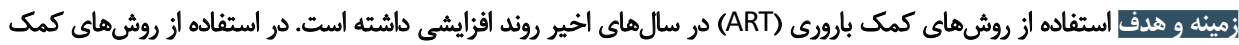

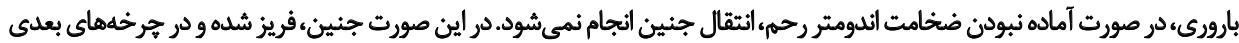

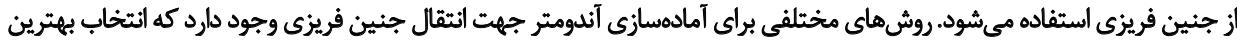

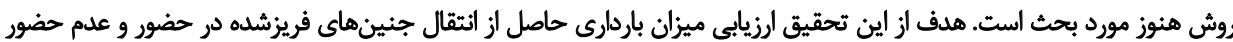

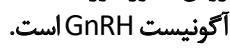

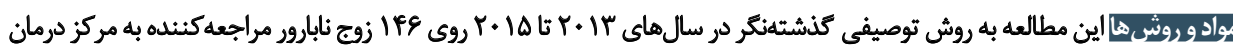

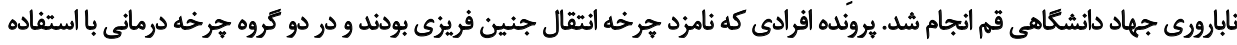

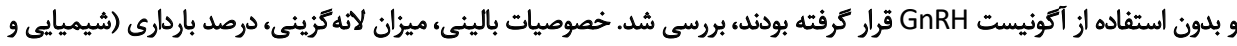

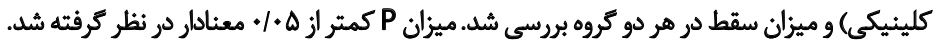

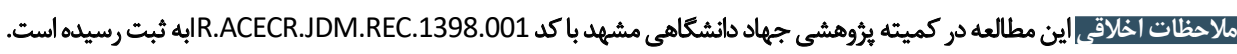

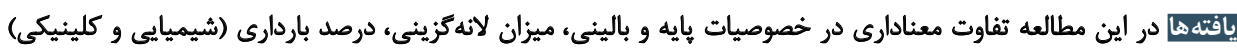

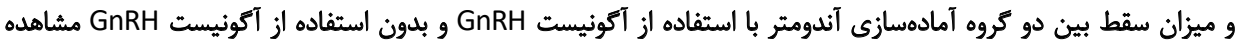
نشد (ه> (P)

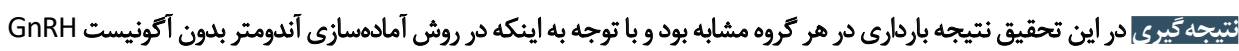

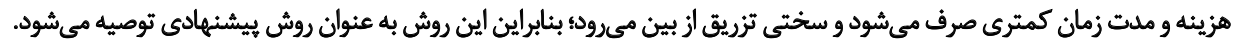

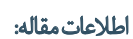

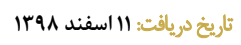

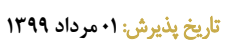
تاريخ انتشار: كا بهمن
\end{abstract}

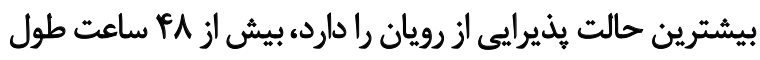

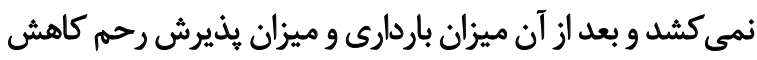

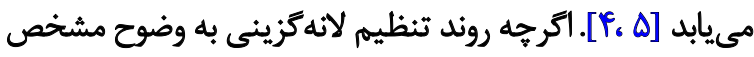

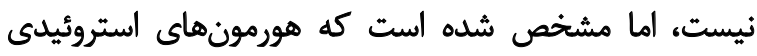

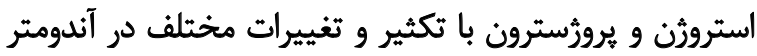

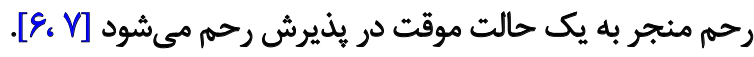

روش هاى مختلفى براى آمادهسازى آندومتر جهت انتقال جنين

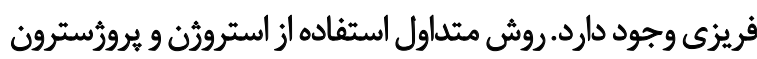

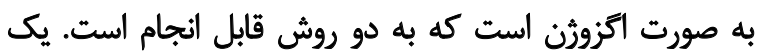

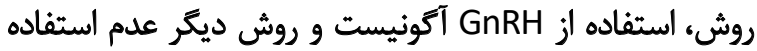

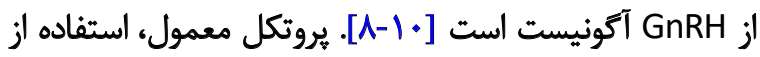

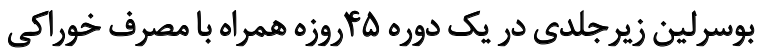

استراديول است (يروتكل استفاده از GnRH) [ [11].

امروزه در روشهاى كمك بارورى' بعد از عمل لقاح گامتهاى

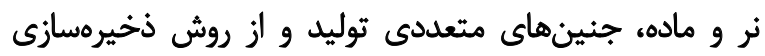

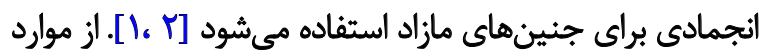

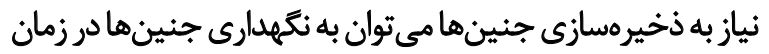

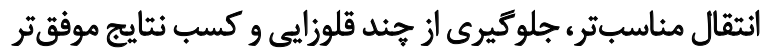

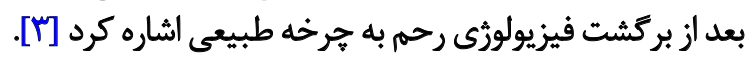

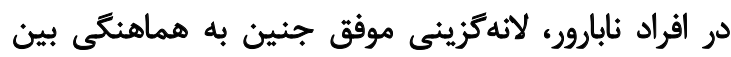

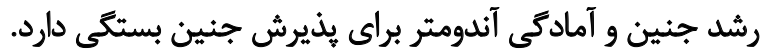

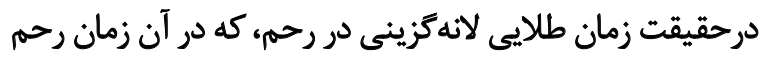

1. Assisted Reproductive Techniques 


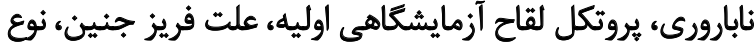

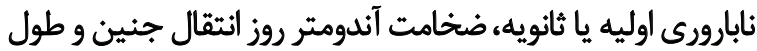

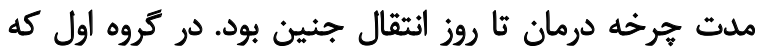

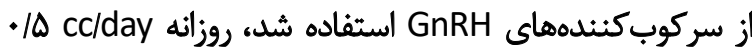

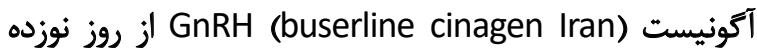

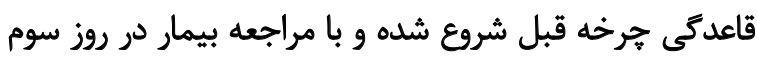

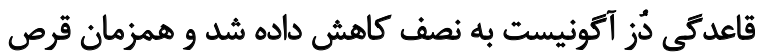

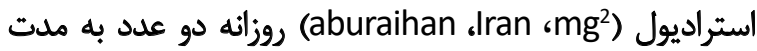

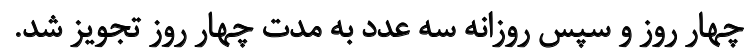

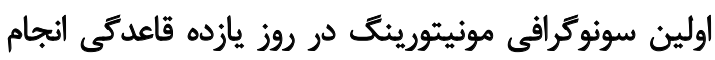

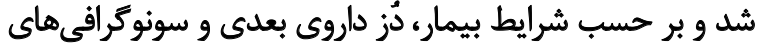

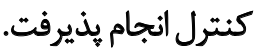

زمانى كه ضخامت آندومتر به Y-9 ميلىمتر ميرسيد، روزانه دور

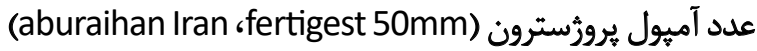
به صورت عضلانى به بيمار طى سه تا بن ينج روز بر حسب شروايط

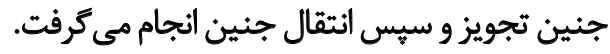

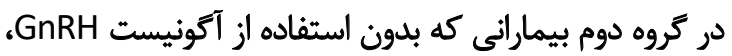

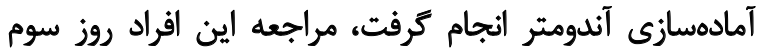

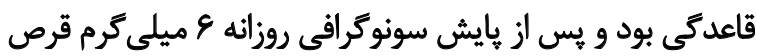

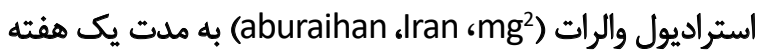

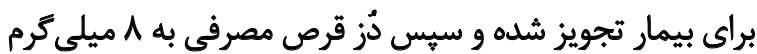

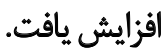

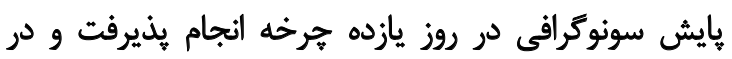

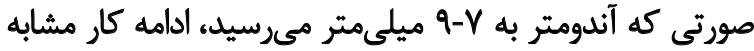

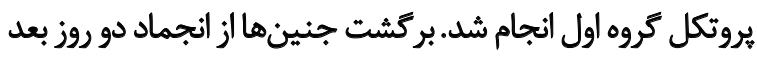

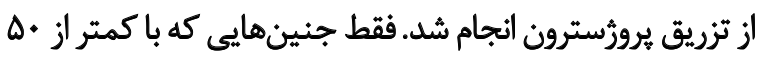
درصد فراتمنتاسيون منتقل شده بودند، مورد مطالعه قرار ترفتيند. انتقال جنين با دستكاه كاتتر لابوتكت ساخت كشور آلمان

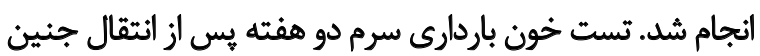

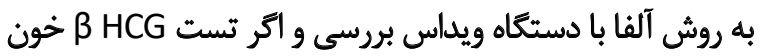

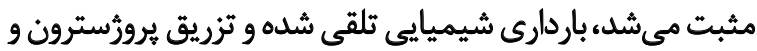

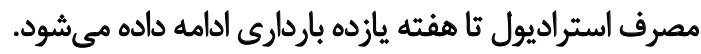
باردارى بالينى با مشاهده ساك حاملكى و مثبت شدن ضربت بانيان

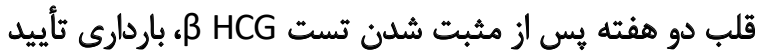

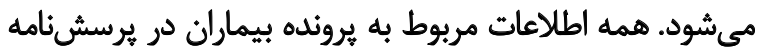

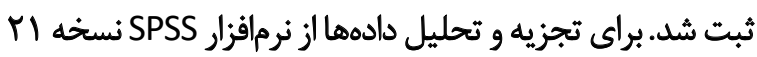

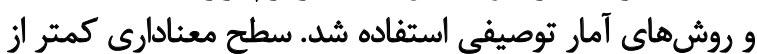

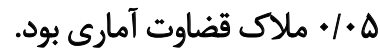

ياقتهها مطالعه حاضر روى |f^| بيمار، كه از جنين فريزشده استفاده
حال با توجه به هزينه تزريق بوسرلين و نيز سختى تزريق،

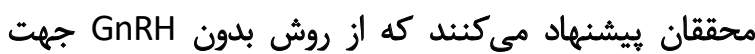

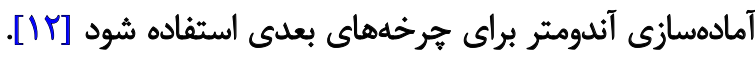

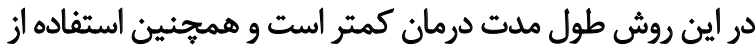

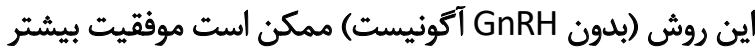

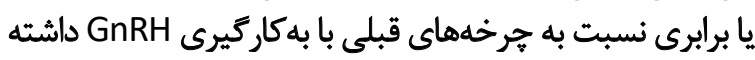

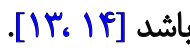

برخى مطالعات، نتايج بهتر را بدون استفاده از آكونيستهاى GnRH

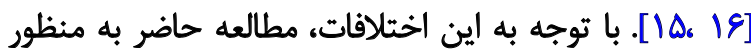

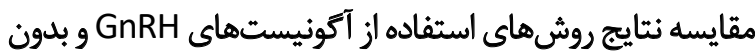
استفاده از آكونيستهاي GnRH براى آمادهسازى آندومتر است.

\section{مواد و روقئها}

اين مطالعه به روش توصيفي كذشتهنكر از بيمارانى كه از سال

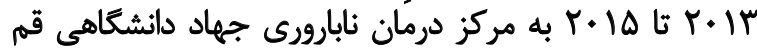

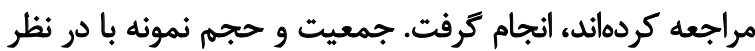
كرفتن سطح اطمينان هو درورد

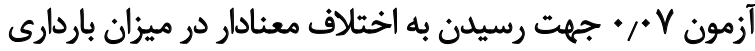

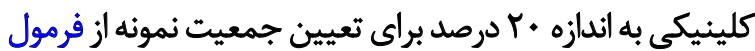

$$
\text { شماره إستفاده شد. }
$$

$$
n=\frac{z^{2} p q}{d^{2}}
$$

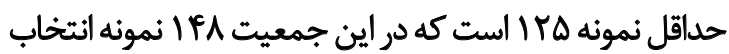

$$
\text { و يرونده افراد بررسى شد. }
$$

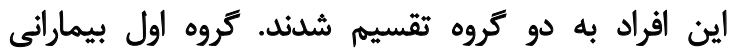

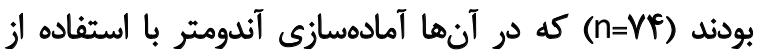

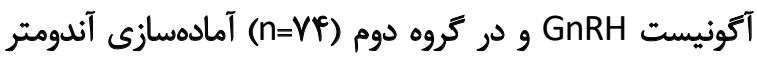

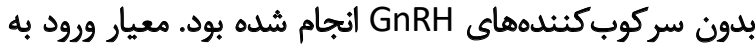

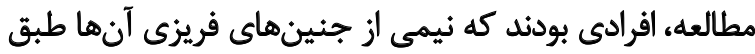

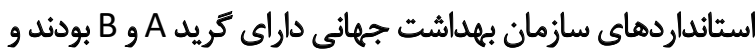

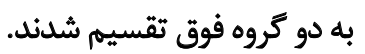

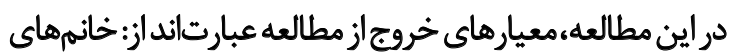

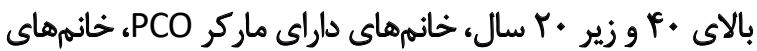

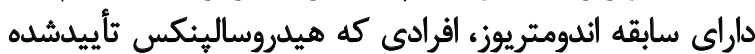

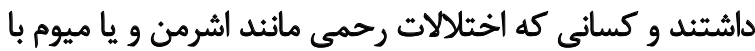

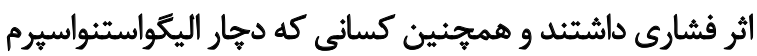
شديل بودند.

جهت دسترسى به مندرجات برونده از بيماران رضايت

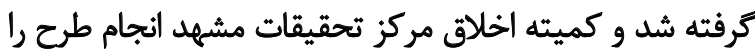

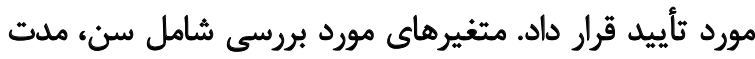


جدول ا. مقايسه خصوصيات بالينى و يايه بين كروه درمان با آكونيست HRnG و بدون درمان با آكونيست HRnG

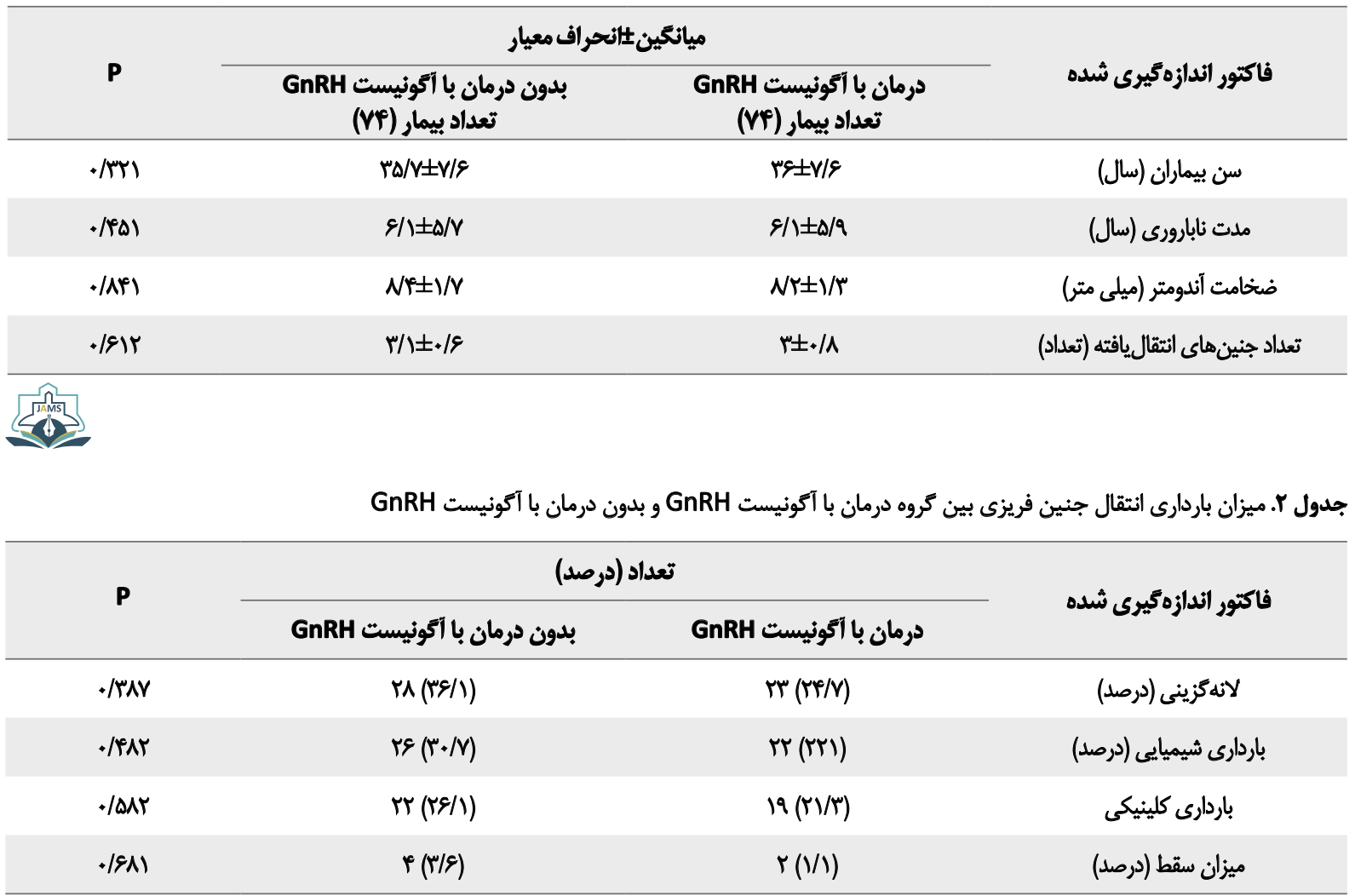

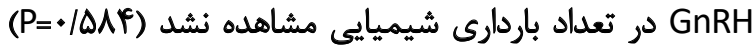

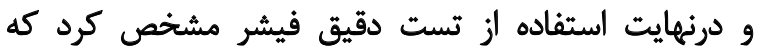

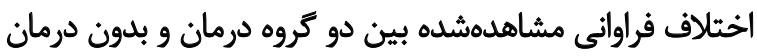

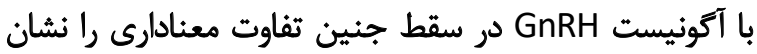

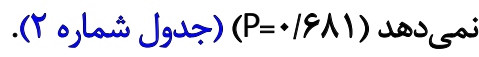

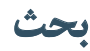

از زمهـان ابــاع روش هاى بارورى آزمايشعاهى سال ها مي كذّرد.

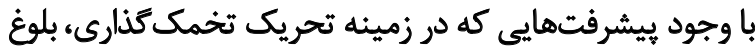

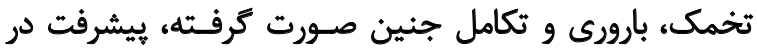

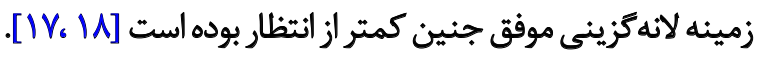

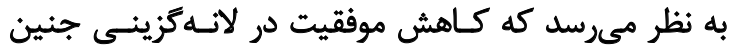

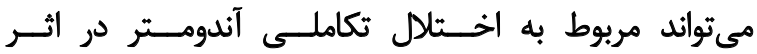

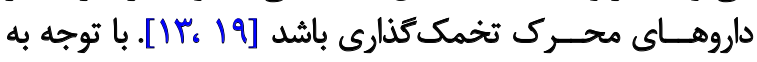

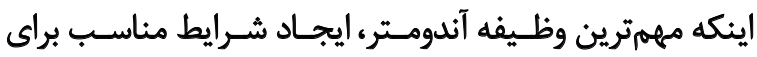

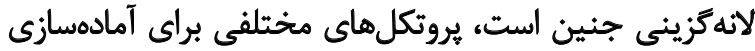

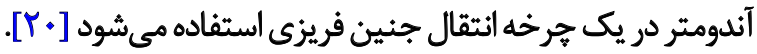

در واقع نتيجه كيرى در هر جرخه انتقال جنينهاي فريز إنداه نياز به هماهنكى دقيق بين آمادهسازى آندومتر و رشد جندين جنين دارد فريز
كرده بودنده انجام شد. آزمون تي مستقل نشان داد كه تفاوت معنادارى بين دو كروه درمان با آتؤيست

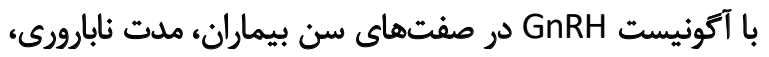

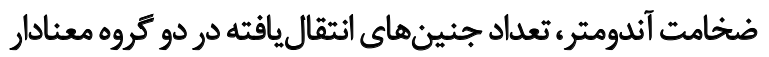

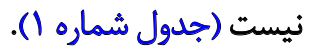
أزمون تى مستقل نشان داد كه اختلاف ميانكين سن بيماران

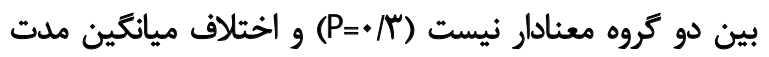

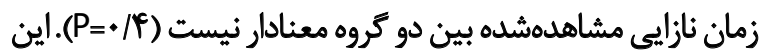

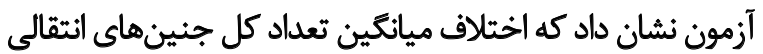

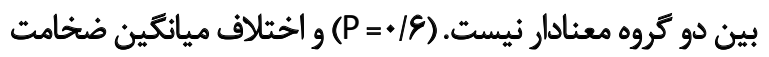

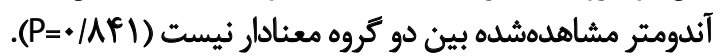

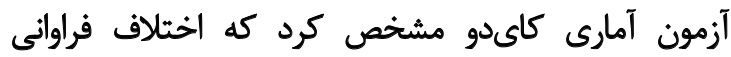

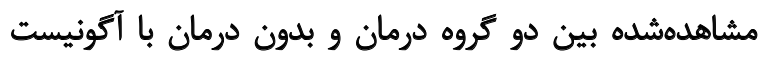
GnRH

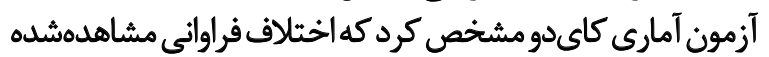

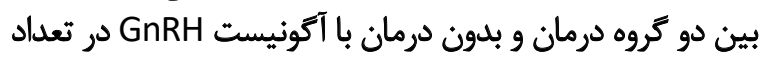

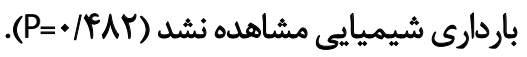

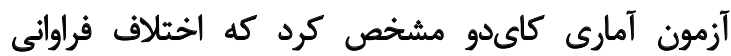

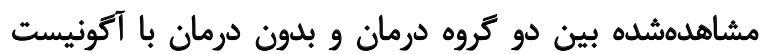


هيل و همكاران كزارش دادند كه وقتى هبروتكل هورمون

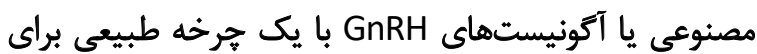

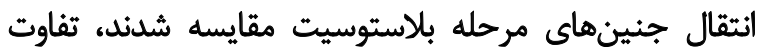

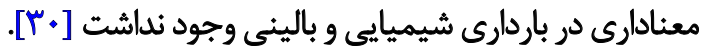
مطالعات فعلى نتايج موفقيتآميز جرخها هادها را بدون استفاده از

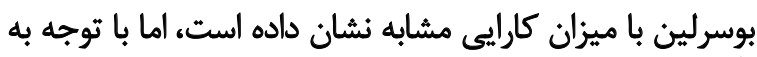

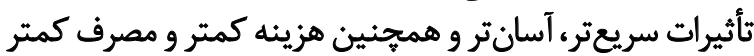

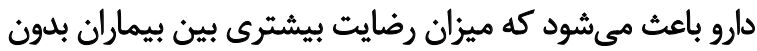
استفاده از GnRH ايجاد شود.

مىتوان نتيجه كرفت كه آمادهسازى آندومتر با استفاده از

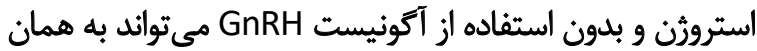

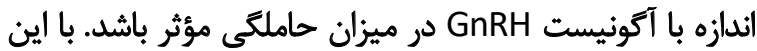

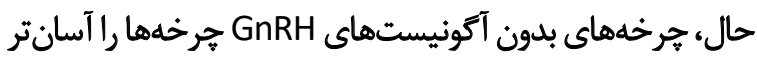

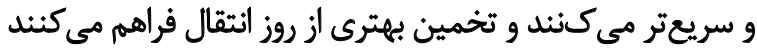

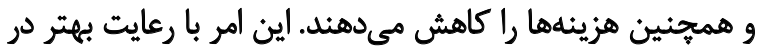
بيماران و ميزان كمتر اثرات سوء دارو همراه است.

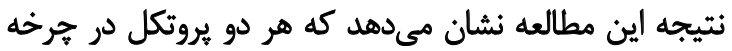

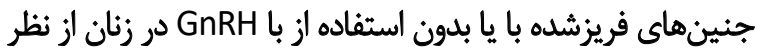

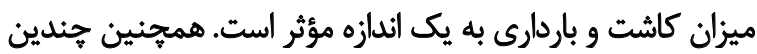

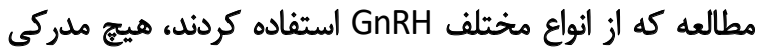

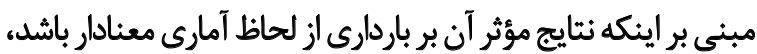

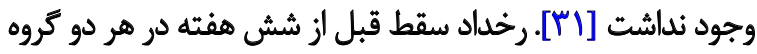

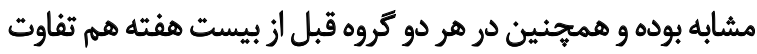

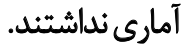

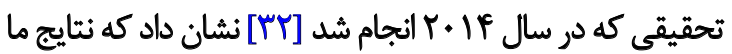

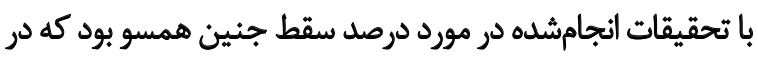
ميزان سقط در هر دو گروه تفاوت معنادارى مشاهده نشد مدرد

\section{تئيجلكَيرى}

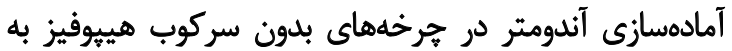

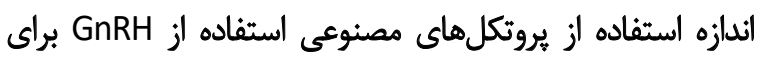

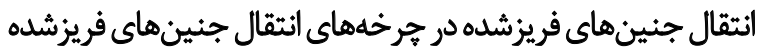

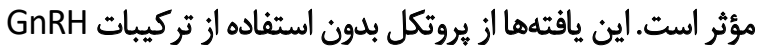

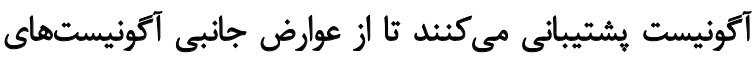

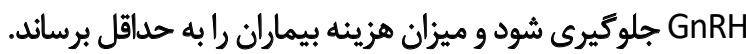

ماحظات اخلاقي - ماتي

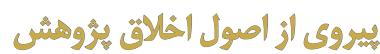

اين مطالعه با بيروى از اصول اخلاق يُروهش در كميثه يروهشى

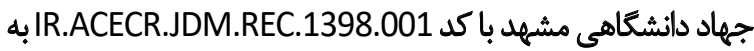

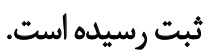

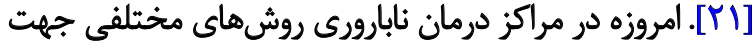
آمادهازي آندومتر جهت انتقال جنين فريزى استفاده مي دشود.

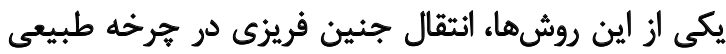

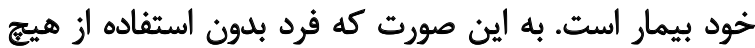

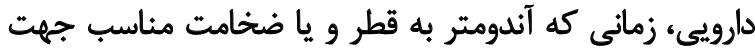

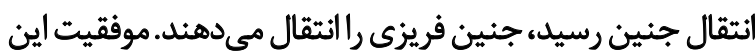

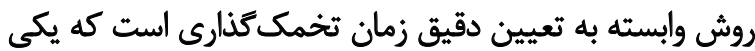

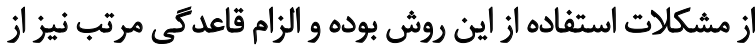

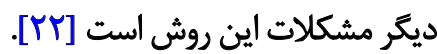

در جرخههاى آمادهازي آندومتر بدون استفاده از آكونيست

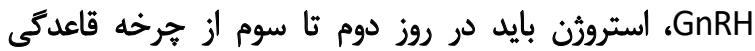

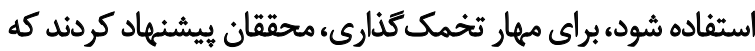

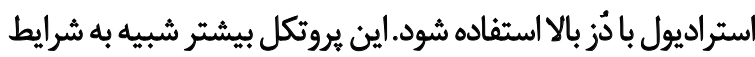

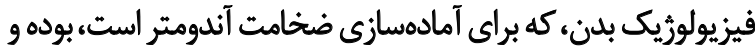

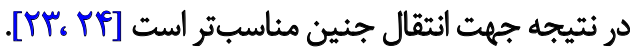

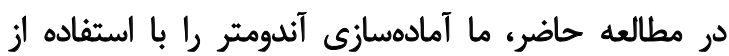

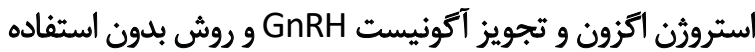

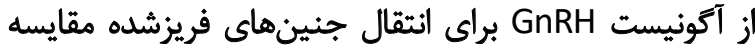

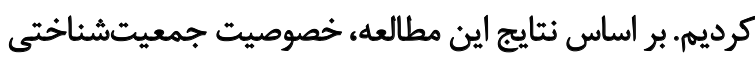
(سن خانم) تفاوت معنادارى را بين دو گروه نشان ندادي

همجنين در اين تحقيق تفاوت معناداري در ضخامت آندومتر

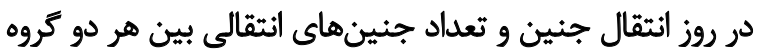
استفاده از آكونيست GnRH و بدون جنديت استفاده از

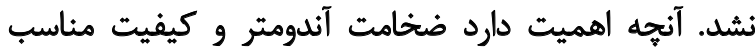

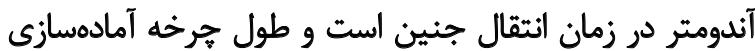
آندومتر ثأثيرى بر نتايج بارورى ندارد.

در مطالعهايى كه الدار جوا و همكاران روى خوى انجام دادندئ

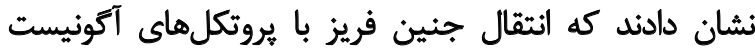

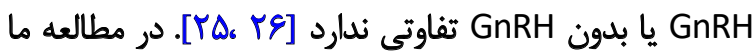

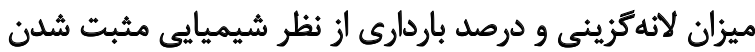

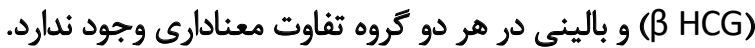

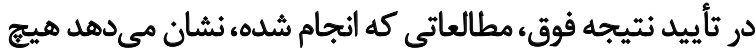

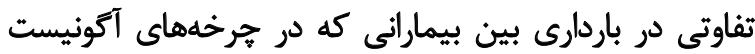

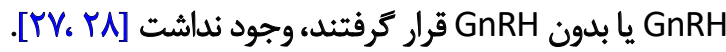
نتايج ما همجنين نشان مى دهد كه جرخ خهماي بدون آكونيست

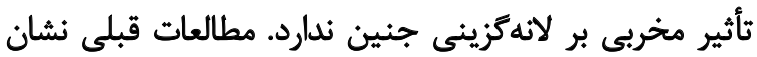

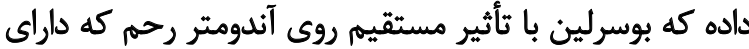

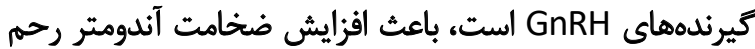

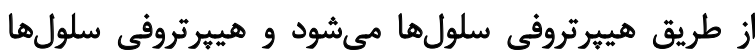

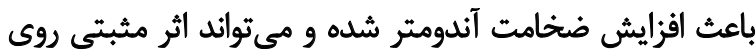

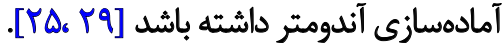




$$
\text { oto sols }
$$

اين طرح با همكارى و هزيئه مركز تخصصى درمان نابارورى جهاد دانشكاهى قهم انجام شده است.

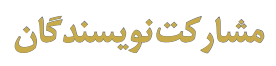

ايده، روش يُروهش و نمونهكيرى: كتايون برجيس، ليلا

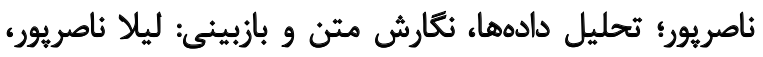
راحيل جنتىفر. تهليل

$$
\text { تعار ض مثأق }
$$

بنابر اظهار نويسندكان اين مقاله تعارض منافع ندارد.

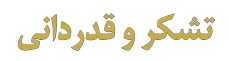

از معاونت ئروهشى مركز جهاد دانشكاهى قمى كه در انجام اين

طرح همكارى كردند، سياس تز زارى مي دشود. 


\section{References}

[1] Oehninger S, Mayer J, Muasher S. Impact of different clinical variables on pregnancy outcome following embryo cryopreservation. Mol Cell Endocrinol. 2000; 169(1-2):73-7. [DOI:10.1016/S03037207(00)00355-5]

[2] Samsami A, Chitsazi Z, Namazi G. Frozen thawed embryo transfer cycles; A comparison of pregnancy outcomes with and without prior pituitary suppression by GnRH agonists: An RCT. Int J Reprod Biomed. 2018; 16(9):587-94. [DOI:10.29252/ijrm.16.9.587] [PMID] [PMCID]

[3] Kassab A, Sabatini L, Tozer A, Zosmer A, Mostafa M, Al-Shawaf T. The correlation between basal serum follicle-stimulating hormone levels before embryo cryopreservation and the clinical outcome of frozen embryo transfers. Fertil Steril. 2009; 92(4):1269-75. [DOI:10.1016/j. fertnstert.2008.08.077] [PMID]

[4] Salumets A, Suikkari AM, Mäkinen S, Karro H, Roos A, Tuuri T. Frozen embryo transfers: Implications of clinical and embryological factors on the pregnancy outcome. Hum Reprod. 2006; 21(9):2368-74. [DOI:10.1093/humrep/del151] [PMID]

[5] El-Toukhy T, Coomarasamy A, Khairy M, Sunkara K, Seed P, Khalaf Y, et al. The relationship between endometrial thickness and outcome of medicated frozen embryo replacement cycles. Fertil Steril. 2008; 89(4):832-9. [DOI:10.1016/j.fertnstert.2007.04.031] [PMID]

[6] Check JH, Dietterich C, Graziano V, Lurie D, Choe JK. Effect of maximal endometrial thickness on outcome after frozen embryo transfer. Fertil Steril. 2004; 81(5):1399-400. [DOI:10.1016/j.fertnstert.2004.01.020] [PMID]

[7] Li S, Li Y. Administration of a GnRH agonist during the luteal phase frozen-thawed embryo transfer cycles: A meta-analysis. Gynecol Endocrinol. 2018; 34(11):920-4. [DOI:10.1080/09513590.2018.148071 4] [PMID]

[8] Orvieto R, Meltzer S, Rabinson J, Zohav E, Anteby EY, Nahum R. GnRH agonist versus $\mathrm{GnRH}$ antagonist in ovarian stimulation: The role of endometrial receptivity. Fertil Steril. 2008; 90(4):1294-6. [DOI:10.1016/j. fertnstert.2007.10.022] [PMID]

[9] Devroey P, Bourgain C, Macklon NS, Fauser BC. Reproductive biology and IVF: Ovarian stimulation and endometrial receptivity. Trends Endocrinol Metab. 2004; 15(2):84-90. [DOI:10.1016/j.tem.2004.01.009] [PMID]

[10] Chau LTM, Tu DK, Lehert P, Dung DV, Thanh LQ, Tuan VM. Clinical pregnancy following $\mathrm{GnRH}$ agonist administration in the luteal phase of fresh or frozen Assisted Reproductive Technology (ART) cycles: Systematic review and meta-analysis. Eur J Obstet Gynecol Reprod Biol X. 2019; 3:100046. [DOI:10.1016/j.eurox.2019.100046] [PMID] [PMCID]

[11] Horcajadas JA, Díaz-Gimeno P, Pellicer A, Simón C. Uterine receptivity and the ramifications of ovarian stimulation on endometrial function. Semin Reprod Med. 2007; 25(6):454-60. [DOI:10.1055/s-2007-991043] [PMID]

[12] Eftekhar M, Dehghani Firouzabadi R, Karimi H, Rahmani E. Outcome of cryopreserved-thawed embryo transfer in the GnRH agonist versus antagonist protocol. Iran J Reprod Med. 2012; 10(4):297-302. [PMID] [PMCID]

[13] Eftekhar M, Rahmani E, Eftekhar T. Effect of adding human chorionic gonadotropin to the endometrial preparation protocol in frozen embryo transfer cycles. Int J Fertil Steril. 2012; 6(3):175-8. [PMID] [PMCID]

[14] Lawrenz B, Samir S, Melado L, Ruiz F, Fatemi HM. Luteal phase serum progesterone levels after $\mathrm{GnRH}$-agonist trigger-how low is still high enough for an ongoing pregnancy? Gynecol Endocrinol. 2018 34(3):195-8. [DOI:10.1080/09513590.2017.1391204] [PMID]

[15] Eldar-Geva T, Zylber-Haran E, Babayof R, Halevy-Shalem T, Ben-Chetrit A, Tsafrir A, et al. Similar outcome for cryopreserved embryo transfer following GnRH-antagonist/GnRH-agonist, GnRH-antagonist/HCG or long protocol ovarian stimulation. Reprod Biomed Online. 2007 14(2):148-54. [DOI:10.1016/S1472-6483(10)60781-X]

[16] Benmachiche A, Benbouhedja S, Zoghmar A, Boularak A, Humaidan P. Impact of mid-luteal phase $\mathrm{GnRH}$ agonist administration on reproductive outcomes in $\mathrm{GnRH}$ agonist-triggered cycles: A randomized controlled trial. Front Endocrinol (Lausanne). 2017; 8:124. [DOI:10.3389/fendo.2017.00124] [PMID] [PMCID]

[17] Rimm AA, Katayama AC, Diaz M, Katayama KP. A meta-analysis of controlled studies comparing major malformation rates in IVF and ICSI infants with naturally conceived children. J Assist Reprod Genet. 2004 21(12):437-43. [DOI:10.1007/s10815-004-8760-8] [PMID] [PMCID]

[18] Mehrafza M, Zare Yousefi T, Saghati Jalali S, Nikpouri Z, Raoufi A, Hosseinzadeh $E$, et al. Comparison of pregnancy outcomes of frozen embryo transfers in women undergoing artificial endometrial preparation with and without short and long-acting gonadotropin releasing hormone agonists. J Midwifery Reprod Health. 2019; 7(4):1929-35. [DOI:10.1007/s10815-004-8760-8]

[19] Basirat, Z, Esmailzadeh S, Jorsaraei, G, Firoozpour M, Abdolhashempour $\mathrm{S}$. [Determining the best appropriate level of endometrial thickness in the outcome of intra-cytoplasmic sperm injection (Persian)]. J Babol Univ Med Sci. 2012; 14 (4):15-21. http://jbums.org/ article-1-4147-en.html

[20] Bourgain C, Devroey P. The endometrium in stimulated cycles for IVF. Hum Reprod Update. 2003; 9(6):515-22. [DOI:10.1093/humupd/ dmg045] [PMID]

[21] Esmailzadeh S, Faramarzi M. Endometrial thickness and pregnancy outcome after intrauterine insemination. Fertil Steril. 2007; 88(2):4327. [DOI:10.1016/j.fertnstert.2006.12.010] [PMID]

[22] Marcondes FK, Bianchi FJ, Tanno AP. Determination of the estrous cycle phases of rats: Some helpful considerations. Braz J Biol. 2002; 62(4A):609-14. [DOI:10.1590/S1519-69842002000400008] [PMID]

[23] Suszka-Świtek A, Czekaj P, Pająk J, Skowronek R, Wrona-Bogus K Plewka D, et al. Morphological and enzymatic changes caused by a long-term treatment of female rats with a low dose of gonadoliberin agonist and antagonist. Med Sci Monit. 2012; 18(8):BR315-330. [DOI:10.12659/MSM.883264] [PMID] [PMCID]

[24] Song M, Liu C, Hu R, Wang F, Huo Z. Administration effects of singledose $\mathrm{GnRH}$ agonist for luteal support in females undertaking IVF/ICS cycles: A metaanalysis of randomized controlled trials. Exp Ther Med. 2020; 19(1):786-96. [DOI:10.3892/etm.2019.8251]

[25] Jelodar G, Gholami S, Jafarpour F. Effect of GnRH on guinea pig endometrium at preimplantation stage. Indian J Exp Biol. 2007; 45:242-6. http://nopr.niscair.res.in/handle/123456789/5682

[26] Blom J, Tan L, Hughes L, Tekpetey F, Rafea BA. Serum estradiol leve on the fifth day of ovarian stimulation in a GnRH antagonist protocol can predict pregnancy outcomes in IVF/ICSI. Fertil Steril. 2018; 110(4):e214. [DOI:10.1016/j.fertnstert.2018.07.618]

[27] Tan SL, Maconochie N, Doyle P, Campbell S, Balen A, Bekir J, et al. Cumulative conception and live-birth rates after in vitro fertilization with and without the use of long, short, and ultrashort regimens of the gonadotropin-releasing hormone agonist buserelin. Am J Obstet Gynecol. 1994; 171(2):513-20. [DOI:10.1016/0002-9378(94)90291-7] 
[28] Bila JS, Vidakovic S, Radjenovic SS, Dokic M, Surlan L, Sparic R. Predictors of IVF/ICSI success following treatment of endometriosis as the cause of primary infertility. Ginekol Pol. 2018; 89(5):240-8. [DOI:10.5603/GP.a2018.0042] [PMID]

[29] Billig H, Furuta I, Hsueh AJ. Gonadotropin-releasing hormone directly induces apoptotic cell death in the rat ovary: Biochemical and in situ detection of deoxyribonucleic acid fragmentation in granulosa cells. Endocrinology. 1994; 134(1):245-52. [DOI:10.1210/endo.134.1.8275940] [PMID]

[30] Hill MJ, Miller KA, Frattarelli JL. A GnRH agonist and exogenous hormone stimulation protocol has a higher live-birth rate than a natural endogenous hormone protocol for frozen-thawed blastocyst-stage embryo transfer cycles: An analysis of 1391 cycles. Fertil Steril. 2010; 93(2):416-22. [DOI:10.1016/j.fertnstert.2008.11.027] [PMID]

[31] Azimi Nekoo E, Chamani M, Shahrokh Tehrani E, Hossein Rashidi B, Davari Tanha F, Kalantari V. Artificial endometrial preparation for frozenthawed embryo transfer with or without pretreatment with depot gonadotropin releasing hormone agonist in women with regular menses. J Family Reprod Health. 2015; 9(1):1-4. [PMID]

[32] Wu Y, Gao X, Lu X, Xi J, Jiang S, Sun Y, et al. Endometrial thickness affects the outcome of in vitro fertilization and embryo transfer in normal responders after GnRH antagonist administration. Reprod Biol Endocrinol. 2014; 12:96. [DOI:10.1186/1477-7827-12-96] [PMID] [PMCID] 